\title{
LE SAVOIR EXPÉRIENTIEL DANS LE CHAMP DE LA SANTÉ MENTALE : LE PROJET EMILIA
}

\author{
Emmanuelle Jouet ${ }^{1}$
}

L'article rend compte de la reconnaissance et de la construction des savoirs des malades au travers d'une recherche-action européenne, Emilia, visant à améliorer l'inclusion sociale des personnes vivant avec un trouble psychique. Dans un premier temps, l'auteur présente les questions de recherche et la méthodologie d'intervention qui ont été mises en place dans le projet Emilia (projet de recherche financé par le sixième cadre de recherche de la Commission européenne (« contrat CIT3513435 »), ainsi qu'une partie des résultats. De 2005 à 2010, le projet Emilia a développé un programme de formation tout au long de la vie et d'accompagnement vers l'emploi dans le milieu ordinaire pour un groupe de personnes vivant avec un trouble psychiatrique. L'étude a analysé les effets de cette intervention basée sur les notions d'empowerment et de Rétablissement sur la qualité de vie et l'utilisation des services sanitaires et sociaux des usagers. Une analyse quantitative a été effectuée à trois instants différents de la recherche (mois 0 , mois 10 et mois 20) au moyen des outils SF36 (Ware et al, 1992) et CSSRI-EU (Client Sociodemographic and Service Receipt Inventory ; Chisholm and Knapp, 2006). Des entretiens qualitatifs (aux mêmes dates) sont venus compléter le recueil de données afin de combiner les résultats quantitatifs et d'évaluer le processus d'insertion

1 EPS Maison Blanche EXPERICE- Université de Paris VIII

Recherches en communication, $\mathrm{n}^{\circ} 32$ (2009). 
professionnelle, sociale et des effets sur la santé des personnes incluses dans la recherche-action. Sur les 35 usagers intégrés à Paris, un tiers d'entre eux ont obtenu un emploi en milieu ordinaire ou participé à des activités de formation qui ont eu un effet positif sur leur qualité de vie et leur utilisation des services sanitaires et sociaux, un tiers ont participé de temps en temps au programme de formation Emilia, un tiers n'a pas modifié son rapport aux services de l'emploi, sanitaires et sociaux.

Dans Emilia, il est reconnu que les personnes vivant avec un trouble psychique possèdent une expérience de la maladie, voire une expertise qui leur permet de faciliter leur qualité de vie et leur inclusion sociale. Au regard de cette posture, sont étudiés quels en ont été les caractéristiques, rôles et fonctions tenus par les usagers tout au long du projet.

Cet article veut rendre compte de la mobilisation de l'expérience de reconnaissance des malades chroniques comme des experts et des co-chercheurs au travers de la recherche-action européenne Emilia (financée par le sixième cadre de recherche de la Commission européenne « contrat CIT3-513435 » portant sur l'inclusion des personnes vivant avec des troubles psychiques.) Pour se faire, après avoir resitué cette question dans le contexte des savoirs des malades, nous verrons quels rôles ont été dévolus aux patients dans le projet et nous en discuterons des limites.

Les lignes de partage entre le savoir des malades et des soignants sont en voie de déplacement dans le système de santé et dans la société. En parallèle d'un patient devenu réformateur (Defert, 1989) qui assume une place décisionnaire dans la démocratie sanitaire, les malades (y compris proches, aidants naturels et patients) revendiquent de plus en plus la possession de savoirs issus de la maladie et mobilisables dans leurs parcours de soins. A l'intérieur du système de soins, les soignants développent des programmes d'éducation thérapeutique afin de former les patients à mieux connaître les symptômes et les traitements, à être davantage observants et à être plus autonomes dans leur vécu avec la maladie (Sandrin- Berthon, 2000, 2001). Corollairement, les malades ont proposé à d'autres malades d'apprendre à partir de leur expérience (peer-support, Alcooliques Anonymes), entre pairs et en revendiquant la possibilité d'autodiriger leurs savoirs. En santé mentale, 
cette dernière voie a donné naissance à différents concepts et pratiques comme le Rétablissement (Recovery, Anthony, 1993, Deegan, 1996), l'empowerment (spécifique à ce champs) (Rapapport, Ahern \& Fisher, 1999 (a) (b)) ou bien Construire sur ses forces (Strength Aproach, Peter Ryan, Paulette Case-Robinson, Peter Sartori \& Peter Jones, Programme de formation Emilia, 2010) où l'expérience de la maladie se positionne comme le socle fondateur de savoirs et de compétences mobilisables par les patients afin d'appréhender leurs soins y compris leur insertion dans la société et ce de façon autre que celle proposée par les services psychiatriques classiques.

Apprendre à l'occasion de la maladie s'inscrit dans une logique de formation et d'éducation tout au long de la vie telle que définie par l'Europe. Il est aujourd'hui admis que se former permet d'améliorer l'état de santé et de bien-être (NIACE, 2010). C'est cette voie, innovante en santé mentale, que la recherche-action européenne Emilia a souhaité explorer. Elle considère les personnes atteintes de troubles psychiques comme experts de leur maladie : au travers d'un programme d'accompagnement à l'emploi et de formations basées sur les principes de l'accès à tous citoyens à l'éducation tout au long de la vie et à l'emploi, d'une part et sur les approches du rétablissement et de l'empowerment d'autre part. A partir du constat que les personnes vivant avec des troubles psychiques sont davantage fragilisées socialement et éloignées de la formation, Emilia cherche à étudier comment, en construisant à partir des forces des usagers et de leur expérience du vécu de la maladie et en développant leur projet professionnel, personnel et de santé, on peut favoriser leur inclusion sociale. Pour aller plus avant, c'est dans le vécu et dans ce qui pourrait être qualifiée d'expertise de ce vécu (cf infra) que les malades vont pouvoir surmonter les obstacles intrinsèques à la maladie mentale, non seulement dans la symptomatologie mais également dans son retentissement au niveau social et professionnel.

\section{Emilia : un projet européen sur l'autonomisation des usagers des services de santé mentale}

Le projet Emilia, Empowerment of Mental Health Service Users through Life Long Learning, Integration and Action (Autonomisation des usagers des services de santé mentale par l'accès à la formation tout au long de la vie et l'intégration active) est un projet de recherche financé par la Commission européenne. Associé à ENTER Mental 
Health - un réseau européen d'établissements publics de santé et d'universités spécialisés dans la formation et la recherche en santé mentale-, le projet Emilia a réuni seize partenaires institutionnels dans douze pays différents. Le site représentant la France était l'Établissement public de santé Maison Blanche à Paris. Le processus général adopté consistait en un développement de programmes de onze formations ad hoc, basés sur les concepts de Recovery et d'Empowerment, que chacun des sites de démonstration proposa à un ensemble d'usagers qui souhaitaient ou retrouver du travail, ou se former, ou participer à une activité significative. Le propos était d'étudier pendant près de deux ans les effets d'une offre de formations et d'un accompagnement à l'emploi sur la qualité de vie et l'utilisation des services sanitaires et sociaux d'usagers souffrant de troubles psychiatriques graves et de longue durée (F20, F31).

En fait, l'objectif d'Emilia consistait à regarder les effets de l'éducation à la formation tout au long de la vie et à l'emploi sur l'inclusion sociale des participants tout en partant de l'idée que l'inclusion devrait se faire via leur mobilisation comme patient expert. C'est de cette mobilisation dont nous voulons particulièrement rendre compte ici et discuter. C'est pourquoi nous présentons ci-après la méthodologie de recherche, les fondements théoriques qui la sous-tendent et les dispositifs de formation développés dans le projet.

L'objectif primaire de la recherche était que l'accès à la formation tout au long de la vie aurait augmenté le nombre de personnes ayant une maladie mentale grave et de longue durée en cours d'emploi.

Les principales hypothèses secondaires étaient que l'accès à la formation tout au long de la vie pour les personnes ayant des maladies mentales graves et de longue durée :

- Aurait augmenté le nombre de jours travaillés pour cette population

- Aurait augmenté le nombre de jours où les personnes sont en formation

- Aurait augmenté le nombre de jours où les personnes se trouvent dans une démarche d'insertion professionnelle

- Aurait augmenté le nombre de jours où les personnes ont des activités de type associatif, bénévole ou salarié, dans la société civile

- Aurait amélioré leur qualité de vie

Par ailleurs : 
- une évaluation qualitative permettrait d'explorer de manière plus large l'effet de l'accès à l'apprentissage tout au long de la vie sur l'inclusion sociale

- une évaluation économique examinerait le coût des résultats du processus de formation et d'insertion sociale.

Une analyse quantitative a été effectuée à trois instants différents de la recherche (mois 0, mois 10 et mois 20) au moyen des outils SF36 (Ware et al, 1992) et CSSRI-EU (Client Sociodemographic and Service Receipt Inventory ; Chisholm and Knapp, 2006). Des entretiens qualitatifs (aux mêmes dates) sont venus compléter le recueil de données afin de combiner les résultats quantitatifs et d'évaluer le processus d'insertion professionnelle, sociale et des effets sur la santé des personnes incluses dans la recherche-action.

\section{Les personnes vivant avec des troubles psychiques sont plus fragiles socialement}

Le projet Emilia est basé sur le Rapport sur l'inclusion sociale 2001 de la Commission européenne, qui part lui-même du constat que l'exclusion sociale réduit sévèrement l'accès aux services et à l'emploi nécessaires à une qualité de vie minimum. Les personnes vivant avec une maladie mentale chronique font partie en effet des personnes les plus exclues parmi les citoyens européens, avec moins de $20 \%$ en cours d'emploi.

De fait, cette population réunit un ensemble de facteurs de vulnérabilité à l'exclusion sociale :

- Si dans la plupart des pays européens, les personnes souffrant de troubles psychiques ont accès aux droits sociaux en termes d'allocations mensuelles, ce revenu ne leur permet de vivre de façon convenable que dans certains pays. (Pour le Royaume-Uni, Ryan et al. 1999) ;

- les personnes ayant un trouble psychiatrique grave ont un niveau d'accès à l'emploi peu élevé. La même étude de Ryan et al (1999) a trouvé que $99 \%$ de la population étudiée était sans emploi et se trouvait dans cette situation depuis plus de trois ans, des résultats confirmés par d'autres études (Burns et al, 1999 ; Thornicroft, 1998);

- au regard de la formation initiale, les personnes ayant un niveau peu élevé d'éducation sont sur-représentées en milieu psychiatrique (European Communities and STAKES, 2004); 
- dans une revue de la littérature sur trouble psychiatrique et handicap, Jenkins et Singh (2001) concluent que « les troubles psychiatriques sont associés à des niveaux élevés de handicap ». De plus, Ryan et al (1998) ont trouvé que 39\% d'une cohorte de personnes présentant un trouble psychiatrique grave avaient aussi des troubles somatiques nécessitant un traitement médical ;

- de nombreuses études décrivent un lien épidémiologique entre le niveau de précarité d'un quartier et la prévalence des troubles psychiatriques les plus courants (Lewis et al, 1998). La précarité du logement est associée à la maladie mentale, avec un nombre significatif de personnes ayant un trouble de santé mentale se trouvant sans domicile fixe (European Communities and STAKES, 2004) ;

- la prévalence d'alcoolisme ou d'abus de substances est élevée chez les personnes présentant un autre trouble psychiatrique, de $30 \%$ à 50\% selon les études (Regier et al, 1995).

\section{Les apprentissages notionnels et méthodologiques sont utiles mais difficiles pour les personnes vivant avec des troubles psychiques.}

La dimension éducative a été également étudiée dans les interventions auprès des personnes vivant avec des troubles psychiques. L'Association Psychologique Américaine (America Psychological Association APA) (1997, in Kopolewicz \& Liberman, 2003) établit que " le rétablissement durable (d'une maladie mentale) nécessite des traitements compréhensifs, coordonnés, consistants, compétents, empathiques et centrés sur la personne pour améliorer les services et les résultats des traitements psychosociaux et pharmaco thérapeutiques. » L'étude de Landsverk \& Kane (1998) montre que les interventions éducatives psychosociales peuvent être bénéfiques dans un large nombre de mesures thérapeutiques et de programmes et peuvent autonomiser les personnes, permettant une meilleure collaboration avec le personnel soignant au sujet de leur traitement et de leur projet de réinsertion. Cette étude insiste également sur l'importance primordiale de fournir des programmes dans lesquels sont inclus :

- des interactions entre pairs (Ascher-Svanum \& Whitesel, 1999); 
- un animateur du groupe avec des aptitudes et des compétences dans la gestion de groupe de personnes vivant avec des troubles psychiques (Hayes \& Gantt, 1992);

- des contenus adaptés, basés sur la résolution de problèmes concrets de tous les jours et sur la réalisation de buts spécifiques et accessibles (Kopolewicz \& Liberman, 2003) et co-construits avec les stagiaires ;

- des formes d'intervention interactives, axées sur les échanges et les discussions davantage que sur une information descendante trop formelle (Emer et al., 2002) ;

- un état d'esprit de groupe positif reposant sur l'espoir et l'encouragement.

Enfin, l'étude conclut sur le fait que les programmes devront proposer aux personnes qui ont le plus de difficultés d'apprentissage, des soutiens individualisés et spécifiques ${ }^{1}$.

On retrouve dans ces recommandations, les éléments qui fondent à la fois l'éducation tout au long de la vie, avec l'utilisation de la formation formelle, mais également informelle et non formelle, ainsi que les concepts de rétablissement, notamment avec ces notions d'Espoir et d'encouragement (Hope). De là, est née l'articulation du projet Emilia entre les objectifs de lutte contre les freins à l'inclusion sociale et l'emploi, et le développement d'un programme d'éducation tout au long de la vie basé sur le rétablissement, l'empowerment et le vécu de la maladie.

\section{Un partenariat d'accompagnement à l'emploi}

Ainsi, les professionnels de santé du $25 \mathrm{e}$ et $7^{\mathrm{e}}$ secteurs psychiatriques de Paris - psychiatres, infirmiers, assistantes sociales, cadres de santé - et les opérateurs de l'accompagnement à l'emploi qui se sont associés au projet - PLIE (Plan local pour l'insertion et l'emploi) du $18 \mathrm{e}$ et $19 \mathrm{e}$ arrondissements de Paris et Maison de l'emploi de Paris (MEP) - ont créé un partenariat afin de proposer des accompagnements spécifiques et de travailler à une pérennisation des actions testées pendant les deux années de la recherche-action. Afin de favoriser la " reprise de pouvoir sur sa vie » (empowerment), il a été proposé à

1 Cette partie a été rédigée par C. Griffith, chercheur dans le projet Emilia. Traduction E.Jouet 
l'usager qui intégrait le programme Emilia d'élaborer et de gérer son projet professionnel, personnel et de santé : accompagné par des professionnels des domaines du médical et de l'insertion professionnelle, et avec le suivi d'un référent parcours Emilia (RPE), l'usager analysait son parcours précédent, identifiait ses forces et se fixait des objectifs à court et moyen termes. Ces derniers pouvaient être tournés vers le retour à l'emploi, la reconversion professionnelle, la formation, mais également des activités significatives, basées notamment sur son « savoir expérientiel » acquis au cours de son parcours au travers de la maladie. Ici intervenaient les référents parcours emploi du PLIE des $18 \mathrm{e}$ et $19 \mathrm{e}$ arrondissements de Paris et les conseillers emploi MEP, ainsi que le RPE qui effectuait régulièrement des entretiens non directifs au téléphone avec les usagers afin de suivre leur parcours et de leur proposer les formations du programme Emilia. Le RPE avait pour fonction d'être un soutien et d'augmenter l'estime de soi des usagers, de réfléchir aux choix effectués, de proposer des solutions avec des informations nouvelles et d'orienter vers des lieux ressources.

Dans la logique d'une organisation apprenante, un comité de pilotage comprenant l'ensemble des parties-prenantes y compris des usagers se réunissait régulièrement afin de déterminer les étapes suivantes du projet. La présence des usagers permettait d'aborder de façon ouverte et transparente des problématiques qui les concernaient directement : faut-il annoncer son handicap psychique ou non lors d'un entretien, pourquoi ne suis-je pas venu au dernier entretien ? les avantages et les inconvénients de la Reconnaissance de la Qualité Travailleur Handicapé, les obstacles et facilitateurs sur le chemin de l'emploi... Développer des liens de connaissance et de confiance entre les services psychiatriques et les services de l'insertion professionnelle au bénéfice de l'usager constituait un pilier des interactions entre les différentes parties-prenantes.

\section{Un programme de formation spécifique à Emilia}

Un panel de formations, destiné aux usagers des services de santé mentale, visant à améliorer la connaissance de la maladie, de ses effets et du « vivre avec », et axé sur le parcours d'insertion professionnelle et sociale, a été élaboré par les partenaires européens. Ces formations sont proposées aux usagers inclus dans la recherche à Paris. 
Liste des formations réalisées au cours de la recherche-action Emilia

- Formation « Intégration » (5 sessions d'avril 2007 à décembre 2007)

- Formation « Gérer son parcours professionnel» (5 sessions)

- Formation « Se rétablir » (janvier 2008)

- Formation « Soutenir un proche » (mai et juin 2007)

- Formation à la recherche - Enquête CLUD, et Ville et Santé mentale - (de novembre 2007 à décembre 2008)

- Formation « Activer son réseau de soutien social» (juin 2008)

- Formation « Prévention du suicide» (novembre 2008)

- formation «Concordance - gestion autonome du traitement » (mars 2009)

- Formation sur la discrimination en psychiatrie (27 avril 2009)

En partenariat

- Formation « Devenir témoin expert des services de santé mentale » (mars 2009) avec la FNAPSY

- Formation «Sensibilisation au handicap psychique » $(8$ sessions de 1 jour entre décembre 2008 et juin 2009) avec la coordination du PDITH

\section{5 co-chercheurs inclus à Paris, 9 emplois dans le secteur ordinaire}

En Europe, dans les huit sites de démonstration qui mettaient en œuvre l'intervention Emilia, près de 216 usagers ont bénéficié du programme local. A Paris, le laboratoire de recherche qui était en charge du projet a recruté 35 usagers. Sur ce total, trente d'entre eux ont effectué au moins une formation (formation initiale), deux, se sont orientés vers le secteur protégé, neuf ont eu accès à un emploi dans le secteur ordinaire et onze ont participé à des activités significatives dans le secteur de la santé mentale.

Un des déterminants du succès de l'approche du rétablissement et de l'empowerment en psychiatrie est la participation des usagers. Ainsi, à Paris des formations hybrides co-construites avec les usagers, ont été conduites. Les bénéficiaires pouvaient être à la fois usagers et proches ou usagers et soignants. L'intérêt d'une telle approche pédagogique est de travailler des notions, comme la prévention du suicide, l'observance du traitement, de façon à rendre la relation soignant/soigné plus symétrique. De plus, les usagers ont été encouragés à élaborer et animer des formations à l'attention des conseillers de Missions locales et autres 
chargés du handicap de grandes entreprises et d'universités sur la maladie, ses causes, ses traitements et ses implications dans le parcours de formation initiale et dans la recherche d'emploi. Ces sensibilisations au handicap psychique étaient ainsi co-animées par un psychiatre et par deux usagers témoins, valorisant et légitimant la parole, l'expérience et le vécu de l'usager.

Les usagers du projet Emilia ont également participé en tant que co-chercheurs à l'intégralité d'un processus de recherche en santé mentale : de la formation à des outils d'enquête (questionnaires, entretiens, enquêtes exploratoires...) à la communication dans des séminaires scientifiques ou des conférences. C'est par exemple le cas d'une recherche sur "Usage de la ville et troubles psychiques 》 réalisée entre les usagers co-chercheurs, le groupe de sociologues, historiens et géographes issus du Collectif «Ville et Santé mentale». Cette recherche a permis d'interroger l'organisation citadine en fonction des pathologies chroniques des chercheurs issus d'Emilia. Le statut de co-chercheur s'inscrit dans les approches de la recherche-action telle que désignée par Lewin (1946) et selon les principes de participation de Freire (1965). Cette figure prend une pleine place dans les recherches-actions qui abordent les thèmes de l'inclusion sociale, de l'insertion professionnelle, de la formation et de la qualité de vie des patients.

De plus, lors de réunions collectives qui évoquaient les obstacles et les facilitateurs dans la réalisation du projet professionnel, personnel et de santé des usagers, les usagers se formaient et acquéraient des savoirs nouveaux dans le triptyque de l'approche de l'autoformation telle que développée par Pineau (1989). Le partage des expériences de chacun avec les autres membres du groupe, le sentiment d'appartenance, l'émulation suscitée par le travail en commun sont autant d'observations réalisées tout au long du projet. 


\section{Eclairage sur la sémantique de l'expertise et de l'expérience}

Pour discuter les résultats du projet Emilia il est utile d'apporter des précisions ${ }^{1}$ sur les lignes de partage du sens des expressions de plus en plus utilisées dans le monde de la santé, comme usager, expert, patient-formateur... Ce ne sont pas uniquement des frontières sémantiques dont il s'agit mais bien de l'émergence d'un nouveau savoir : l'expérience de la maladie comme source de savoirs et qui a été la trame de la conceptualisation du programme éducatif proposé dans Emilia.

\section{En quoi le patient peut-il être expert?}

L'invocation du qualificatif « expérientiel » apparaît en 1998 à propos du diabète puis d'autres maladies chroniques (en particulier en rhumatologie), en parallèle avec la notion de patient expert introduite, elle, aux Etats-Unis dès 1985. Dans ces expressions, la référence à l'expert peut être comprise de plusieurs façons complémentaires : une personne ayant une expertise dans un domaine, reconnue par la société, et une personne vivant une expérience, qu'elle soit subie (l'expérience de la vie, l'expérience de la maladie), choisie (faire l'expérience de la vie à l'étranger), voire même décidée pour vérifier des hypothèses (au sens de la méthode expérimentale de Claude Bernard).

\section{L'expert qui apporte une expertise reconnue}

Étymologiquement, le mot « expert » vient du latin classique expertus, qui veut dire « éprouvé, qui a fait ses preuves $» .^{2}$ Un deuxième sens proche renvoie à des « composés désignant un professionnel ou un

1 Nous reprenons ici de courts extraits de la note de synthèse (Jouet, Flora, Las Vergnas, 2010) parue dans le numéro 57/58 (janvier -juin 2010), co-dirigé par l'auteur, de la revue Pratiques de formation : Analyses, Saint Denis, Université Paris 8, consacré à la question de « la part des savoirs des malades dans le système de santé ».

2 L'adjectif « expert à, en, dans... » se réfère à « une personne, à ses activités, à ses gestes et désigne quelqu'un qui a acquis une grande habileté, un grand savoir-faire dans une profession, une discipline, grâce à une longue expérience ». Le substantif masculin qualifie un "spécialiste habilité auprès d'un tribunal ou d'une instance quelconque à émettre un avis sur une question exigeant des connaissances spéciales » et par extension un « spécialiste, connaisseur d'un domaine particulier». 
spécialiste agréé auprès d'une instance ». Dans cette seconde catégorie se regroupe l'ensemble des experts appelés par la Loi pour analyser, évaluer, donner un avis et statuer sur une situation où des tiers sont en opposition ou risquent de l'être. L'expertise revêt ici un caractère technique et promeut les caractéristiques d'une personne qui dispose de compétences et de connaissances techniques sur un sujet et qui est habilité de par la Loi à rendre un avis. Les experts médicaux, judiciaires, en assurances, sont autant de figures rencontrées couramment dans la vie quotidienne.

De fait, cette acception correspond à l'expertise mobilisée dans la plupart des nouvelles postures et figures décrites plus haut. Le pair aidant, le patient formateur constituent autant d'experts au sens de spécialistes reconnus. Au figuratif, expertiser devient d'ailleurs « examiner avec acuité, avec insistance » et il arrive que l'on fasse appel à cette intervention d'examen dans le cadre des missions des représentants des usagers dans les conseils d'administration et autres instances des hôpitaux, comme le Comité de lutte contre les infections nosocomiales (Clin), le Comité de liaison alimentation nutrition (Clan), le Comité de lutte contre la douleur (Clud).

\section{L'expert qui vit son expérience}

Le patient expert prend de fait une autre coloration si on le rattache plus spécifiquement à son " expérience », qui est un « fait vécu » qui consiste à « acquérir, volontairement ou non, ou à développer la connaissance des êtres et des choses par leur pratique et par une confrontation plus ou moins longue de soi avec le monde $»^{1}$. L' « expérience» devient alors aussi le " résultat de cette acquisition, l'ensemble des connaissances concrètes acquises par l'usage et le contact avec la réalité de la vie, et prêtes à être mises en pratique ».

\section{Un expert dont l'expérience de la maladie devient savoir}

Ce n'est donc pas dans un «processus expérimental» mais bien par une «formation expérientielle » que le vécu « in vivo » et même

1 Plus globalement, elle est même « d'une manière générale et abstraite, l'instruction acquise par l'usage de la vie ». Sachant que par extension, on a donné dans un sens concret le nom « d'expérience » aux faits qui nous fournissent cette instruction expérimentale des choses, ce qui réfère à la méthode de Claude Bernard. 
plutôt « in situ » d'une maladie se métamorphose en savoirs et qu'un malade se transforme en expert ${ }^{1}$.

\section{Patience, expérience et savoirs}

Un patient dont l'expertise est reconnue et vient de l'expérience de la maladie. Ainsi, aujourd'hui, les conceptions d'un patient capable d'expertise d'une part, et d'un patient ayant vécu l'expérience de la maladie d'autre part, se rejoignent grâce à la reconnaissance des savoirs expérientiels liés à la maladie. De fait, le patient est de plus en plus souvent reconnu et utilisé comme expert ayant acquis son expertise par l'expérience. Voilà qui rejoint la signification philosophique de l'expérience qui s'affirme comme une « connaissance acquise soit par les sens, soit par l'intelligence, soit par les deux, et s'opposant à la connaissance innée impliquée par la nature de l'esprit ». En la matière, reprenant les thèses de Laing (1973), Rogers (1969, 1976), Illich (1971) et Dewey (1968), Bézille (2003) repère les lignes de partage entre une expérience vécue et une expérience formatrice (organisée au plan didactique), une expérience formative (auto-contrôlée), une expérience construite (distanciée). Ces frontières sont pertinentes dans la métamorphose entre un patient et un patient expert (Vallée et al., 2008).

\section{L'expertise en action}

Dans Emilia, les patients ont mobilisé, notamment, ces différentes dimensions de l'expert. Sur les trente-cinq usagers intégrés, un tiers d'entre eux ont obtenu un emploi en milieu ordinaire ou participé à des activités de formation qui ont eu un effet positif sur leur qualité de vie et leur utilisation des services sanitaires et sociaux, un tiers ont participé

1 La formation expérientielle consiste en ce que " les adultes se forment aussi en dehors de lieux et de systèmes de formation institués, en vivant des expériences, [et elle est ainsi] le produit d'expériences qui n'ont pas comme finalité première la formation » (Bonvalot, 1991, p.321) et comme le rappelle R. Massé, " la maladie peut [...] être vue comme un syndrome d'expériences typiques » « $\mathrm{La}$ maladie peut donc être vue comme un syndrome d'expériences typiques », soit un ensemble d'unités de significations ancrées dans l'histoire de vie et attribuées à des évènements critiques (traumatismes personnels, antécédents de maladies, chômage, crises conjugales), à des expériences thérapeutiques, aux réactions de l'entourage et aux émotions (craintes, attentes, anxiété) ». Raymond Massé dans le Dictionnaire du corps, « Maladie » (p. 539-542). 
de temps en temps au programme de formation Emilia, un tiers n'a pas modifié son rapport aux services de l'emploi, sanitaires et sociaux.

$\mathrm{Au}$ cœur du projet Emilia se trouve cette posture d'un patient expert et participant : les programmes de formations qui ont été développés dans l'ensemble des huit sites de démonstration et adaptés au cadre culturel français ont été conçus par des groupes d'usagers formateurs spécialisés. Le module « Devenir co-chercheur » est l'adaptation d'un cours d'usagers qui enseignent à l'Université de Middlesex sur les théories et méthodologies de recherche (Research Skills: a training programme for people who use mental health services, Janet Holmshaw, Kate Holmes, Jacqui Lynskey, programme Emilia). Le module « Prévention du suicide » a été élaboré par un groupe d'usagers professeurs et de psychiatres qui travaillent à la Clinique psychiatrique de l'Hôpital universitaire de Vilnius, qui ont une longue histoire dans ces problématiques de la dépression et du suicide auprès des soignants et des usagers. Au niveau local, les usagers ont fait partie du comité de pilotage d'Emilia, côtoyant ainsi l'ensemble des professionnels parties-prenantes de l'intervention. Du côté de la formation, ils ont été actifs dans l'adaptation des programmes de formation ainsi que dans leur évaluation. Les éléments notionnels étaient revus pour les sessions suivantes ainsi que les exercices et les modalités de mise en œuvre de la formation, qui venaient transformer les programmes initiaux. De plus, certains d'entre eux ont été recrutés comme formateurs et intégrés dans l'ensemble du dispositif de formations, pour participer à des enquêtes dans les services psychiatriques, rôles dévolus habituellement aux infirmiers. Toutes ces activités ont eu un impact sur la culture des professionnels, en travaillant notamment les représentations sur les capacités d'apprentissage et de réalisation de soi à l'encontre des personnes vivant avec des troubles bi-polaires ou schizophrènes. Ici, la formule de l'organisation apprenante (Birleson, 2001) a pris toute sa valeur : il s'agit de faire participer l'ensemble des partenaires à ce processus professionnels du sanitaire et du social, usagers, proches, employeurs, collègues - et d'évaluer l'impact sur l'ensemble des participants et non pas uniquement sur les usagers. Le lieu de soins devient aussi un lieu d'apprentissage, d'activité, voire d'emploi.

Sont ainsi identifiables les figures de l'expertise, dans les différents rôles et fonctions tenus par les participants à Emilia. Cependant, il est à noter qu'en raison du mode de fonctionnement des projets européens, les usagers ne pouvaient intervenir en direct dans le protocole 
de recherche et sa gouvernance qui par construction est antérieur à l'arrivée des usagers.

On peut ainsi évoquer une nouvelle approche du soin, où soutien par les pairs, «vivre avec » la maladie (le concept de rétablissement), l'autonomie (empowerment) deviennent les piliers du projet formalisé par l'usager. L'empowerment est déterminé par le niveau d'influence qu'une personne peut exercer sur les évènements de sa vie. Fitzsimmons \& Fuller définissent les opérations suivantes comme étant les résultats de la reprise de pouvoir sur sa vie, qui se sont retrouvées tout au long du processus Emilia :

- un sens du contrôle et d'autodétermination sur les objectifs et les circonstances qui importent pour les personnes ;

- un sens d'auto-efficacité ou de confiance en soi d'être capable de réaliser ces projets ;

- l'augmentation de son niveau d'acceptation de soi-même et de valeur de soi-même ;

- un sens d'être valorisé et respecté par les autres;

- un sens des buts et d'avancement dans la vie selon ses propres intérêts ;

- une vision de la vie positive et motivée.

Emilia a transformé les usagers en co-chercheurs, co-formés et co-formateurs. Et, en considérant que le malade peut non seulement tenir de manière pertinente un rôle de formateur (au sens de pair aidant, Provencher et al., 2010), mais aussi celui de co-chercheur, Emilia s'est inscrit dans un courant qui postule que l'expérience du vécu de la maladie chronique permet de développer un savoir propre, légitimement transférable (dès lors qu'il est étayé et partagé) et qu'il s'inscrit en complémentarité de celui développé académiquement. Les co-chercheurs peuvent non seulement être médiateurs des savoirs médicaux mais aussi producteurs de savoirs créés par une forme de travail de clinique sur leurs propres troubles et protocoles comme c'est le cas pour d'autres pathologies chroniques, telles que la fibromyalgie. 


\section{Références}

Abram, K., \& Teplin, L. (1991). Co-occurring disorders amongst mentally ill jail detainees: implications for public policy. American Psychologist; 46, 1036-1045.

Ahern, L., \& Fisher, D. (1999) (a). People can and do recover from mental illness, NEC Newsletter, Lawrence, MA.

Ahern, L., \& Fisher, D. (1999). (b). PACE, a Recovery Guide, Lawrence, MA.

Anthony, W. (1993). Recovery from mental illness: the guiding vision of the mental health service system in the 1990s, Academic Search Premier. Psychosocial Rehabilitation Journal, 16 (4), 11-23.

Ascher-Svanum, H. \& Whitesel, J. (1999). A randomized controlled study of two styles of group patient education about schizophrenia. Psychiatric Services, 50, 926-930.

Bernard, C. (1947). Principes de médecine expérimentale, fragments rédigés entre 1858 et 1877. Paris : Les Presses Universitaires de France.

Birleson, P. (1998). Learning organisations: a suitable model for improving mental health services? Australian and New Zealand Journal of Psychiatry, 2 (32), 214222.

Bézille, H. (2003). L'autodidacte - Entre pratiques et représentations sociales. Paris : L'Harmattan.

Bonvalot, G. (1991). Éléments d'une définition de la formation expérientielle. In La formation expérientielle des adultes. Paris : La Documentation française, 317-325.

Burns, T. et al., (1999). Intensive versus standard case management for severe psychotic illness: a randomised trial. The Lancet, 353, 2185-2189.

Carré, P. (2005). L'Apprenance, Vers un nouveau rapport au savoir. Paris : Dunod.

Carré, P., Fenouillet, F. (2009). Traité de psychologie de la motivation. Paris : Dunod.

Commission of the European Communities (2001) Joint Report on Social Inclusion.

Cuffel, B., et al., (1994). A longitudinal study of substance misuse and community violence in schizophrenia. Journal of Nervous and Mental Disease, 182, 704-708 .

Deegan, P. (1996). Recovery as a Journey of the Heart. Psychiatric Rehabilitation Journal, 19(3), 91-97.

Defert, D. (1989). Cinquième conférence international sur le Sida, Montréal.

Dewey, J. (1968). Expérience et éducation (Experience et education, 1938). Paris : Armand Colin.

Emer, D., McLarney, A., Goodwin, M. \& Keller, P. (2002) Which group teaching styles best promote information gain for adults with mental disorders? Journal for Specialists in Group Work, 27, 205 - 232.

Feldman, J., et Le Grand, J-L. (1996). Savoirs savants, savoirs profanes. In Feldman, J., Filloux, J.C., Lécuyer, B.P., Slez, M., Vicente, M. (Eds). Ethique, Epistémologie et sciences de l'homme. Paris: L'Harmattan, 89-105.

Freire, P. (1971). La pédagogie des opprimés, Paris : Maspéro (première publication en portugais, 1968).

Jouet, E., Flora, L. \& Las Vergnas, O. (2010). « Construction et Reconnaissance des savoirs expérientiels ». Note de synthèse du $n^{\circ} 57 / 58$, Pratique de formation : Analyses, Saint Denis, Université Paris 8, pp 13-94. 
Haywood, T. et al., (1995). Predicting the revolving door phenomenon among patients with schizophrenic, schizo-affective and affective disorders. American Journal of Psychiatry. 152, 856-861.

Holmshaw, J. et al., (2010). Research Skills: a training programme for people who use mental health services, programme Emilia .

Illich, I. (1971). Une société sans école (titre original : Deschooling Society). Paris : Seuil.

Jenkins, R., \& Singh, B. (2001). Mental disorder and disability in the population. In Thornicroft, G., \& Szmukler, G. Textbook of Community Psychiatry. Oxford. 1011116.

Kaes, R. (1979). Introduction à l'analyse transitionnelle. In Kaes, R., et al. Crise, rupture et dépassement, Paris : Dunod, 1-81.

Kopelowicz, A., \& Liberman, R. P. (2003). Integration of care: integrating treatment with rehabilitation for persons with major mental illnesses. Psychiatric Services, 54, 1491-1498.

Laing, R. (1969). La politique de l'expérience. Paris : Stock.

Landsverk, S. S., \& Kane, C. F. (1998). Antonovsky's sense of coherence: theoretical basis of psychoeducation in schizophrenia. Issues in Mental Health Nursing, 19, 419-431.

Las Vergnas, O. (2007). La culture scientifique et technique comme idéologie. Alliages, $59,20-28$.

Le Boterf, G. (1981). L'enquête participation en question, Paris : Ligue française de l'enseignement et de l'éducation permanente. En ligne : http://openlibrary. org/b/OL3866916M/enquel̀?te_participation_en_question [dernière consultation 30/12/09].

Lewis, G., \& Booth, M. (1994). Are cities bad for your mental health? Psychological Medicine, 24, 913-916.

Lewis, G, et al., (1998). Socio-economic status, standards of living and neurotic disorder, The Lancet, 352, 605-609.

Lewin, K. (1946). Action research and minority problems. J Soc Issues, 2, 34-46.

NIACE (2010). Lifelong learning: contributing to wellbeing and prosperity.

Pineau, G. (1989). La formation expérientielle entre auto, éco et coformation, In Courtois B. (Ed.), Éducation Permanente, 100-101, 23-30.

Provencher, H., et al., (2010). Quand le savoir expérientiel influence nos pratiques. In Jouet, E., et Flora, L. (Eds). Usagers-experts : la part du savoir des malades dans le système de santé, Pratiques de Formation/Analyses, 57/58, 155-174 .

Pouchelle, M-C. (2003). L'hôpital corps et âme. Essais d'anthropologie hospitalière. Paris : Seli Arslan.

Rappaport, J (1984). Studies in Empowerment: introduction to the Issues. Prevention in Human Services, 3, 1-17.

Regier, D., et al., (1995). Co-morbidity of mental disorders with alcohol and other drug abuse, JAMA, 264, 2511-2518 .

Rogers, C. (éd.) (1969). Le développement de la Personne, Paris : Dunod. Première édition (1961). On Becoming a Person: A Therapist's View of Psychotherapy. Boston: Houghton Mifflin.

Rogers, C. (1976). Liberté pour apprendre, Paris : Dunod. Première édition (1969). Freedom to learn: A view of what education might become. Columbus, $\mathrm{OH}$ : Charles Merrill. 
Ryan, P., Ford, R., Beadsmoore, A., \& Muijen, M., (1999). The Enduring Relevance of Case Management, BJ Social Work, 29, 97-125.

Ryan, P., Case-Robinson, P., et al., (2010). Strengh Approach, Emilia programme.

Rappaport, J. (1990). Research methods and the empowerment agenda. In Tolan, P., et al., (Eds). Researching Community Psychology. Washington DC American Psychological Association .

Pečiulis, S., et al (2010). Education program for suicide prevention among mental health care users, programme Emilia.

Sandrin-Berthon, B. (2000). "Pourquoi parler d'éducation dans le champ de la medicine ?". L'éducation du patient au secours de la médecine. Paris : PUF, 7-39.

Sandrin-Berthon, B., et al (2001). L'éducation pour la santé. Un enjeu pour le système de santé. Paris : Ed.CFES.

STAKES \& European Commission (2000) Public Health Approach to Mental Health in Europe.

Swofford, C. et al., (1996). Substance use: a powerful predictor of relapse in schizophrenia, Schizophrenia Research, 20, 145-151 .

Syrot, P. (2009). Épisodes et sociabilité autodidactiques. Pour une description compréhensive des relations sociales du sujet en situation d'information. Thèse en Sciences de l'éducation, Centre de Recherche en Éducation et Formation, Université de Nanterre.

Thornicroft, G. (1998). The PRiSM Psychosis Study articles 1-10, British Journal of Psychiatry, 173, 363-431.

Vallée, J-P., Drahic, E., LeNoc, Y. (2008). « Médecine générale : de quels experts avons-nous besoin ? ", Médecine, 4, 33-40.

Wils, J. (2002). « Les relations avec les usagers », Revue hospitalière de France, 487, 13-16. 